\title{
PSEUDOAINHUM IN A CASE OF PITYRIASIS RUBRA PILARIS- A RARE CASE REPORT
}

\author{
Rajalakshmi R', Seethalakshmi R. S2, Balasubramanian $N^{3}$
}

${ }^{1}$ Assistant Professor, Department of Dermatology, Chennai Medical College Hospital and Research Centre, Irungalur, Trichy, Tamilnadu, (The Tamilnadu Dr. M.G.R. Medical University, Chennai).

2Senior Resident, Department of Dermatology, Chennai Medical College Hospital and Research Centre, Irungalur, Trichy, Tamilnadu, (The Tamilnadu Dr. M.G.R. Medical university, Chennai).

${ }^{3}$ Professor, Department of Dermatology, Chennai Medical College Hospital and Research Centre, Irungalur, Trichy, Tamilnadu, (The Tamilnadu Dr. M.G.R. Medical University, Chennai).

\section{ABSTRACT}

\section{BACKGROUND}

Constriction bands around the digits are termed as ainhum; those which mimic ainhum are termed as pseudoainhum. Ainhum and pseudoainhum can cause autoamputation of the digits. Ainhum which is of idiopathic origin is common in Negros, but rare in rest of the world. Many hypotheses were promulgated for the aetiology of pseudoainhum; however, it has been associated with hereditary palmoplantar keratodermas, systemic sclerosis, leprosy, psoriasis, vascular anomalies etc. We report a case of pseudoainhum associated with pityriasis rubra pilaris (PRP), which has not been reported in the literature so far.

\section{KEYWORDS}

Pseudoainhum, Ainhum, Autoamputation, Pityriasis Rubra Pilaris.

HOW TO CITE THIS ARTICLE: Rajalakshmi R, Seethalakshmi RS, Balasubramanian N. Pseudoainhum in a case of pityriasis rubra pilaris- A rare case report. J. Evolution Med. Dent. Sci. 2017;6(30):2478-2480, DOI: 10.14260/Jemds/2017/535

\section{BACKGROUND}

Ainhum is an idiopathic disease involving the fifth toe of Black people mainly in tropical zones, whereas pseudoainhum is a rare congenital or acquired disorder clinically characterised by progressive development of a fibrotic band on a finger or toe until spontaneous autoamputation occurs. Various aetiological hypotheses are notified which include infections (Mycosis, mycobacteria), trauma (Plants), vascular (dysplasia), neurological (Polyneuritis), and genetic (keratoderma) mechanisms. ${ }^{1}$ Pseudoainhum has been reported in association with hereditary palmoplantar keratodermas, systemic sclerosis, leprosy, psoriasis, vascular anomalies, etc. We report a case of pseudoainhum associated with pityriasis rubra pilaris, which has not been reported in the literature so far.

\section{CASE REPORT}

A 43-year-old married male farmer, born to consanguineous parents, presented to us with itchy, pigmented, hyperkeratotic follicular papules, thickening of skin over both palms and soles since childhood. He gave a history of spontaneous loss of $5^{\text {th }}$ toe of the right foot, following a constriction ring at the base of the same toe. There was mildly pigmented thickened skin over distal part of left lower extremity with a constriction involving the fifth toe. Patient was not a smoker. There was no history of drug intake for any other systemic illness. There was no history of trauma to the feet.

Financial or Other, Competing Interest: None.

Submission 06-03-2017, Peer Review 25-03-2017,

Acceptance 05-04-2017, Published 13-04-2017.

Corresponding Author:

Dr. Rajalakshmi. R,

Assistant Professor

Department of Dermatology,

Chennai Medical College Hospital and

Research Centre, Irungalur,

Trichy-621105, Tamilnadu.

E-mail: rajiijipmer@yahoo.co.in

DOI: $10.14260 /$ jemds $/ 2017 / 535$
There was no history of any pain or discomfort in the affected limb and the patient was able to perform his normal daily activities. Patient gave a history of similar hyperkeratotic skin lesions over the trunk and both extremities in his ancestors, and few of his maternal relatives, but there was no history of autoamputation of toes in any of his family members. General and systemic evaluation was within normal limits. Cutaneous examination revealed multiple discrete follicular hyperkeratotic papules distributed over the extensor and flexor aspects of both forearms (Figure 1), both legs, dilated follicular orifices over dorsa of both wrists, fingers (especially over the knuckles) (Figure 2), legs, feet (Figure 3), and toes. There was symmetrical thickening of skin over both palms and soles with a yellowish discolouration (PRP sandal) (Figure 4). There was loss of $5^{\text {th }}$ toe in the right foot (Figure 5). There was a constriction band around the base of the right $4^{\text {th }}$ and left $5^{\text {th }}$ toes. Nails and oral mucosa were normal. Peripheral arterial pulses were felt equally on both extremities. There were no sensory alterations in any limbs nor was there any abnormality in sweating. Routine haematological, biochemical including thyroid function tests, serological tests including HIV were within normal limits. ENT, dental and ophthalmological assessment was done but no significant abnormalities were detected. Ultrasonogram of the abdomen and pelvis showed a normal study. Punch biopsy was done from hyperkeratotic papules with prominent follicular openings and sent for histopathological examination. There was marked orthokeratosis, focal parakeratosis, papillomatosis, acanthosis and follicular plugging with focal hypergranulosis. Dermis showed sparse superficial perivascular lymphocytic infiltration. All the histopathological features were consistent with pityriasis rubra pilaris. After checking the fasting lipid profile, the patient was started on Cap. Isotretinoin $10 \mathrm{mg}$ daily along with topical emollients and keratolytics. After three weeks, there was significant improvement of follicular hyperkeratosis and keratoderma of both palms and soles. Patient is still under regular follow-up. 


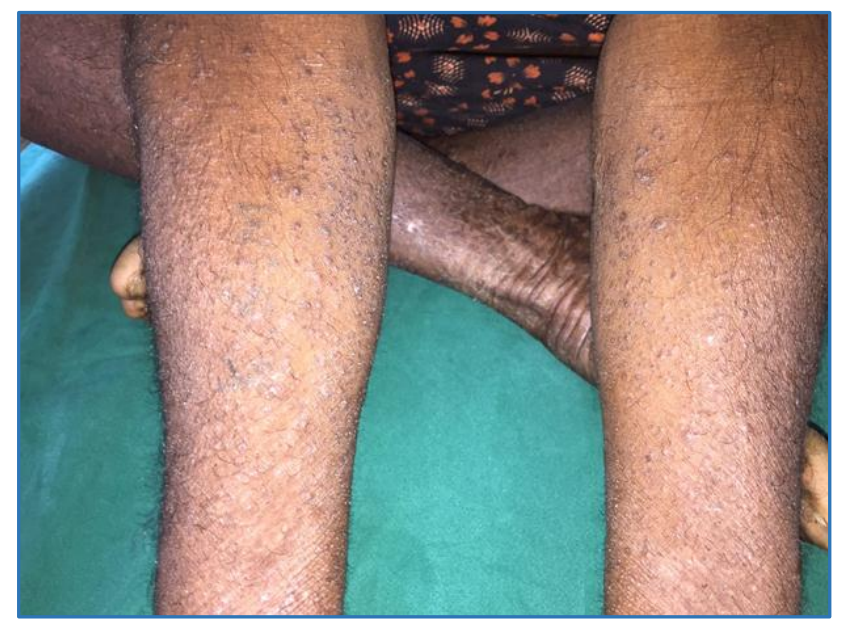

Figure 1. Multiple Discrete Follicular Hyperkeratotic Papules Distributed over Both Forearms

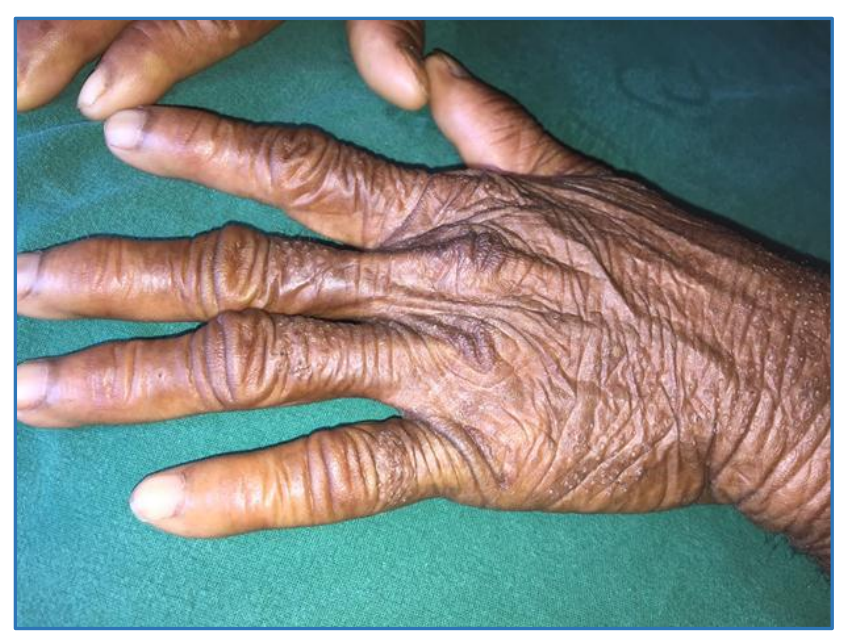

Figure 2. Prominent Follicular Orifices over Wrists, Fingers

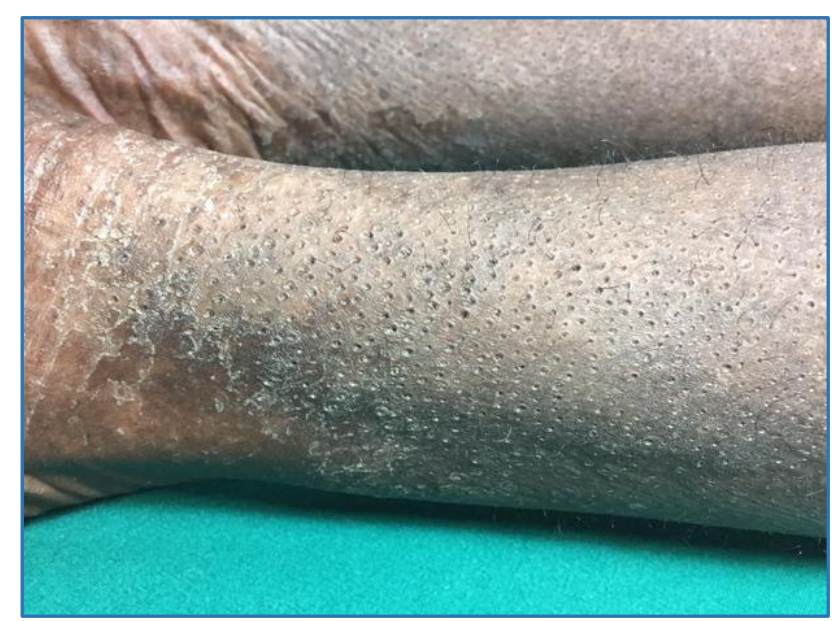

Figure 3. Prominent Follicular Orifices over Legs

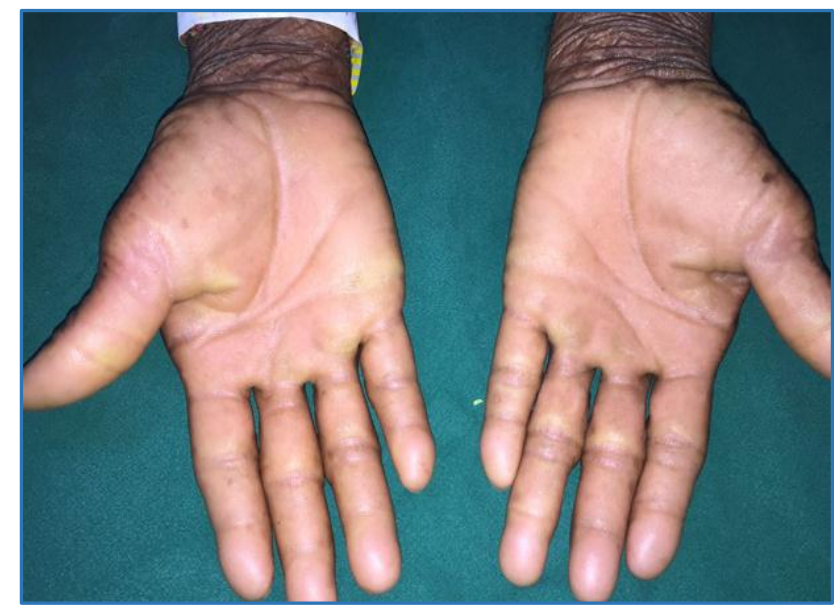

Figure 4. Symmetrical Keratoderma of Both Palms.

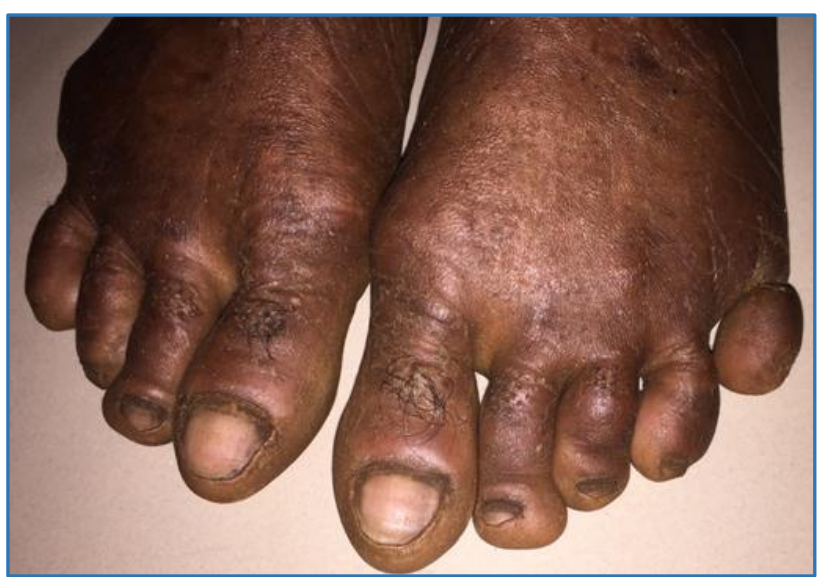

Figure 5. Pseudoainhum- Auto-amputated Right $5^{\text {th }}$ Toe, Constriction at the Base of Left $4^{\text {th }}$ and $5^{\text {th }}$ Toes

\section{DISCUSSION}

Constricting bands are classified as ainhum and pseudoainhum. Ainhum describes the development of constricting bands around toes in underdeveloped countries of Africa and may ultimately result in autoamputation. In the African Yoruba language, ainhum means "to saw" or "file" and in the Brazilian patois, it means "fissure." In the remainder of the world, constricting bands that mimic ainhum are termed pseudoainhum. In the tropical and subtropical climates, the incidence of ainhum has been reported as between $0.015 \%$ and $2 \%$ of the population. This same condition is rarely seen throughout the rest of the world. Pseudoainhum of all types is very rare throughout the world.(2)

The pathogenesis of ainhum has not been clearly elucidated. Chronic trauma, infection, hyperkeratosis, decreased vascular supply, and impaired sensation may produce excessive fibroplasia in a susceptible host. There are three pathophysiological categories of pseudoainhum- (1) congenital constricting bands are caused by the umbilical cord; (2) constriction by external forces, such as hairs or threads, which are generally factitial; and (3) constricting bands secondary to other diseases. These may be hereditary or nonhereditary. 
Hereditary causes include pachyonychia congenita, Mal de Meleda, Unna-Thost, Vohwinkel, Papillon-Lefevre syndrome, $(3,4)$ mutilating keratoderma, lamellar ichthyosis. Nonhereditary diseases include vascular anomalies as seen in Raynaud disease, diabetes mellitus, linear scleroderma, systemic sclerosis and psoriasis. $(5,6,7,8)$ Sensory changes associated with leprosy, tertiary syphilis, syringomyelia, and spinal cord tumours as well as trauma resulting in scar formation from burns, frostbite, and physical trauma can also cause constricting bands to form.(2) Few other mechanisms have also been proposed for pseudoainhum, like infections (Mycosis, mycobacteria), trauma (Plants).(1) Pseudoainhum can be treated with topical pimecrolimus, systemic retinoids like acitretin, and surgeries like excision of the constricting band and Z- plasty.

Pityriasis rubra pilaris refers to a group of chronic disorders characterised by reddish orange plaques with pityriasiform scaling showing follicular keratoses, palmoplantar keratoderma, and sometimes erythroderma. Familial as well as acquired forms of the disease have been reported.(9) Pityriasis rubra pilaris (PRP) has been reported in association with autoimmune disorders like Grave's disease,(10) HIV infection, internal malignancies, Streptococcal infections, hypothyroidism, systemic sclerosis.(11) Generalised cystic acne like lesions, elongated follicular plugs, lichen spinulosus like lesions may be present in patients with PRP associated with HIV.(12) Kaposi's varicelliform eruption has also been reported in association with PRP.(13) So far pseudoainhum has not been reported in patients with PRP. Our report substantiates the association of PRP and pseudoainhum. Although it is a rare association, early diagnosis and intensive medical and surgical management may avoid progression and autoamputation of digits.

\section{CONCLUSION}

Pseudoainhum is a rare clinical entity of varied causes including genetic, acquired, vascular, trauma, etc. The pseudoainhum does not cause any mortality but autoamputation causes disfigurement and discomfort to the patient in walking and other day-to-day activities. Among the disorders of keratinisation, pseudoainhum has been reported in association with psoriasis so far. Literature search did not reveal any cases of pseudoainhum in association with PRP, so far.

\section{REFERENCES}

[1] Morand JJ, Lightburn E. Ainhum and pseudoainhum: clinical aspects and etiopathogenic hypotheses. Med Trop (Mars) 2002;62(2):166-70.

[2] Brodell RT, Helms SE. Ainhum and pseudoainhum. In: Goldsmith LA, Katz SI, Gilchrest BA, et al. (eds) Fitzpatrick's Dermatology in General Medicine. $8^{\text {th }}$ edn. McGraw Hill.

[3] Mashhood AA, Humayun A, Saleem M, et al. PapillonLefevre syndrome associated with pseudoainhum. J Am Acad Dermatol 2004;51(2 Suppl):S134-6.

[4] Ashwani P, Swapna K, Rani SM, et al. Papillon-Lefevre syndrome with pseudoainhum. Indian Dermatol Online J 2010;1(1):33-5.

[5] Kumar P, Gandhi V. Pseudoainhum in psoriasis. Indian J Dermatol 2012;57(3):238-9.

[6] McLaurin CL. Psoriasis presenting with pseudoainhum. J Am Acad Dermatol 1982;7(1):130-2.

[7] Almond SL, Curley RK, Feldberg L. Pseudoainhum in chronic psoriasis. Br J Dermatol 2003;149(5):1064-6.

[8] Ahn SJ, Oh SH, Chang SE, et al. A case of infantile psoriasis with pseudoainhum successfully treated with topical pimecrolimus and low-dose narrowband UVB phototherapy. J Eur Acad Dermatol Venereol 2006;20(10):1332-4.

[9] Sehgal VN, Srivastava G. (Juvenile) pityriasis rubra pilaris. Int J Dermatol 2006;45(4):438-46.

[10] Elamin Abdelgadir EI, Bashier AMK, Makeen SAM, et al. Type II pityriasis rubra pilaris associated with Grave's disease-case report. J Clin Exp Dermatol Res 2015;6:6.

[11] Frikha F, Frigui M, Masmoudi H, et al. Systemic sclerosis in a patient with pityriasis rubra pilaris. Pan Afr Med J 2010;6:6.

[12] Pavithran K, Karunakaran M, Palit A, et al. Disorders of keratinization. In: Valia RG, Valia AR, (eds). IADVL Textbook of Dermatology. $3^{\text {rd }}$ edn. Bhalani Publishing House, Mumbai. 2012:955-1069.

[13] Ng SK, Ang CB, Tham A. Kaposi's varicelliform eruption in a patient with pityriasis rubra pilaris. J Am Acad Dermatol 1992;27(2 Pt 1):263. 